\title{
Exosomes derived from preadipocytes improve osteogenic differentiation, potentially via reduced miR-223 expression
}

\author{
WENHUA DU ${ }^{1,2^{*}}$, LINGKAI SU $^{3^{*}}$, NING ZHANG $^{4}$ and HUIMING WANG ${ }^{1}$ \\ ${ }^{1}$ Department of Oral Implantology, Stomatology Hospital, School of Medicine, Zhejiang University, Hangzhou, \\ Zhejiang 310058; ${ }^{2}$ Department of Oral Implantology and General Oral Health, Bybo Dental Group, Hangzhou, \\ Zhejiang 310004; ${ }^{3}$ Department of Conservative Dentistry and Endodontics, Stomatology Hospital, \\ School of Medicine, Zhejiang University, Hangzhou, Zhejiang 310058; ${ }^{4}$ Stomatology Department, \\ Meitan General Hospital, Beijing 100088, P.R. China
}

Received May 26, 2018; Accepted November 13, 2018

DOI: $10.3892 / \mathrm{mmr} .2018 .9760$

\begin{abstract}
Insufficient bone volume remains a key issue when using dental implants. Adipose tissue-derived stem cells (ADSCs) can accelerate bone healing when combined with dental implants. To improve the application of ADSCs for dental uses, the present study aimed to identify optimal implantation conditions. Mesenchymal stem cell-derived exosomes can induce naïve stem cells to differentiate through the osteogenic lineage. In the present study, exosomes derived from 3T3L1 preadipocytes (3T3L1-exo) were purified and characterized. The effects and potential mechanisms of 3T3L1-exo on 3T3L1 cell ossification were examined by reverse transcription-quantitative polymerase chain reaction, western blotting, electron microscopy, RNA sequencing and histological analysis. The current study confirmed that 3T3L1-exo enhanced 3T3L1 preadipocyte osteogenic differentiation, as revealed by upregulation of osteogenic differentiation-associated genes and increased Alizarin Red staining. Furthermore, the microRNA ( $\mathrm{miR}$ ) expression profiles of 3T3L1-exo and 3T3L1 preadipocytes were sequenced and compared. The results of a further analysis demonstrated that miR-223 expression was reduced in 3T3L1 preadipocytes stimulated by 3T3L1-exo compared with in unstimulated cells. This finding suggested that 3T3L1-exo promoted 3T3L1 bone formation by decreasing miR-223 through a competitive mechanism, another miRNA, or another factor. The mechanism by which miR-223 is decreased warrants further investigation.
\end{abstract}

Correspondence to: Professor Huiming Wang, Department of Oral Implantology, Stomatology Hospital, School of Medicine, Zhejiang University, 866 Yu-Hang-Tang Road, Hangzhou, Zhejiang 310058, P.R. China

E-mail: hmwang1960@hotmail.com

*Contributed equally

Key words: exosome, microRNA, preadipocyte, miR-223, osteogenic differentiation
In conclusion, the application of 3T3L1-exo may be useful for investigating preadipocyte-induced bone regeneration.

\section{Introduction}

Dental implantation has been carried out for the last 25 years and involves placing artificial tooth roots into the jaw to hold a replacement tooth or bridge following tooth loss. The success of long-term dental implant placement relies on essential interactions between the jawbone and dental implant (1). However, insufficient bone volume has a serious impact on these interactions. Furthermore, bone loss following implantation is another major issue to address. Consequently, bone regeneration may require stimulation prior to implantation for a successful outcome (2). To resolve deficiencies in jawbone regeneration and to prevent subsequent bone loss, researchers and dentists have applied numerous strategies, including the use of autografts, xenografts, allografts and alloplastic materials. However, limitations exist for these approaches, including limited availability of grafting material for autografts, and morbidity and insufficient osteogenesis of xenografts, allografts and alloplastic materials, due to an absence of cell populations $(2,3)$.

Cell transplantation technologies may address the limitations of bone transplantation (2). The application of stem or stromal cells offers a promising approach for enhancing osseointegration $(1,4)$. Mesenchymal stem cells (MSCs) can differentiate into bone, adipose, cartilage, muscle and ligament cells. Bone marrow-derived MSCs (BMSCs) can be immunoselected from bone marrow and culture-expanded. BMSCs are one of the most widely applied stem cells for bone regeneration, including that of the jawbone. However, the application of BMSCs for osteogenesis has limitations since cell numbers are insufficiently high for several clinical indications, and the steps for their harvesting and expansion are complex (1). Adipose tissue-derived stem cells (ADSCs) result in bone formation comparable to that of BMSCs and address many of the limitations of BMSCs. Adipose tissue is an attractive MSC source because it is readily accessible by routine liposuction with minimal morbidity (5). Furthermore, a higher number of stem cells can be harvested from adipose 
tissue. ADSCs have been reported to accelerate bone healing in combination with dental implants (1); however, drawbacks to cell-based therapies include risk of tumor and the formation of emboli (6).

Recent evidence has suggested that the secreted factors released by MSCs are more beneficial in tissue regeneration than their direct tissue intercalation and differentiation (7). Additionally, MSCs, including ADSCs, produce exosomes that serve a role in several biological functions. Exosomes are nanovesicles ranging between 30 and $100 \mathrm{~nm}$ in size, which are derived from numerous cell types (8). They act as cell-to-cell messengers and contain mRNA, microRNA (miRNA/miR), proteins and lipids, all of which influence cell fate. Exosomes are considered a novel alternative to stimulate bone regeneration with fewer safety considerations, by resolving the risks of toxicity, emboli, tumorigenicity and immunogenicity (9). Furthermore, they have powerful pro-osteogenic potential (6) and very high stability, in they can be maintained for $\sim 6$ months in vitro at $-20^{\circ} \mathrm{C}$ without loss of potency (10).

MSC-derived exosomes (MSC-exo) can induce naïve stem cells to differentiate through the osteogenic lineage (11). Additionally, mineralized osteoblast (12), dendritic (13) and monocyte cell-derived exosomes have been reported to increase MSC osteogenic differentiation (14). Over the past 3-4 years, exosomes, in particular MSC-exo, have gained prominence in research on bone regenerative medicine (11). However, MSC-exo have the same limitations as MSCs with regards to resource and quality.

Although immortalization compromises the differentiation potential of the MSCs, it does not affect the production or quality of the exosomes for therapy (15). Consequently, ADSC-derived exosomes (ADSC-exo) may represent a more promising tool for bone regeneration. ADSC-exo has been reported to improve osteogenesis via the promotion of vessel formation (16); however, the effects of these exosomes on ADSC osteogenesis are unclear. The present study identified a murine preadipocyte cell line, 3T3L1 cells, as having MSC-like functions and an ability to differentiate into osteoblasts in response to stimulation with differentiation factors. Subsequently, 3T3L1 cell-derived exosomes (3T3L1-exo) were generated, and were used to determine their effects on 3T3L1 cell osteogenesis and to identify a possible mechanism of action. The results revealed that 3T3L1-exo promoted 3T3L1 preadipocytes to undergo osteogenic differentiation via reduced miR-223 expression. Based on these findings, 3T3L1-exo may represent a useful tool for investigating preadipocyte-induced bone regeneration.

\section{Materials and methods}

Cell culture. The 3T3L1 murine preadipocyte cell line was obtained from the American Type Culture Collection (Manassas, VA, USA) and cells were maintained in Dulbecco's modified Eagle's medium (DMEM: high-glucose, 4,500 mg/l; Gibco; Thermo Fisher Scientific, Inc., Waltham, MA, USA) supplemented with $10 \%$ fetal calf serum (Gibco; Thermo Fisher Scientific, Inc.) at $37^{\circ} \mathrm{C}$ with $5 \% \mathrm{CO}_{2}$ For preparation of exosomes, 3T3L1 cells were seeded to $80 \%$ confluence in $100-\mathrm{mm}$ tissue culture dishes. Cells were then cultured for 2 days in the presence of medium containing $2 \%$ exosome-free serum (obtained by ultracentrifugation of serum at $100,000 \mathrm{x} \mathrm{g}$ for $14 \mathrm{~h}$ at $4^{\circ} \mathrm{C}$ ).

Isolation of exosomes. In accordance with a previously reported method (17), cell culture supernatants were collected and centrifuged at $300 \mathrm{x} \mathrm{g}$ for $10 \mathrm{~min}, 1,200 \mathrm{x} \mathrm{g}$ for $20 \mathrm{~min}$, and $10,000 \mathrm{x} \mathrm{g}$ for $30 \mathrm{~min}$ at $4^{\circ} \mathrm{C}$. The supernatant from the final centrifugation was then ultracentrifuged at $100,000 \mathrm{x} \mathrm{g}$ for $1 \mathrm{~h}$ at $4^{\circ} \mathrm{C}$. After removing the supernatant, the exosome pellets were washed in a large volume of ice-cold PBS and centrifuged at $100,000 \mathrm{x}$ for another $1 \mathrm{~h}$ at $4^{\circ} \mathrm{C}(17)$.

Detection of $3 T 3 L 1$ cell proliferation and apoptosis. To determine the effects of 3T3L1-exo on 3T3L1 cell survival, cell apoptosis and proliferation assays were performed. Briefly, cells were stimulated with $2 \mu \mathrm{g} / \mathrm{ml} 3 \mathrm{~T} 3 \mathrm{~L} 1$-exo for $24 \mathrm{~h}$ prior to analysis of the degree of apoptosis or proliferation at $37^{\circ} \mathrm{C}$ with $5 \% \mathrm{CO}_{2}$. For the detection of apoptosis, $2 \times 10^{5}$ cells were stained with fluorescein isothiocyanate-Annexin V (BD Pharmingen; BD Biosciences, San Diego, CA, USA) and propidium iodide (Sigma-Aldrich; Merck KGaA, Darmstadt, Germany) for $5 \mathrm{~min}$ at $4^{\circ} \mathrm{C}$ in the dark. The cells were then analyzed by fluorescence-activated cell sorting to identify positively stained apoptotic cells, as described previously (17).

For the detection of proliferation, $10 \mu$ l Cell Counting kit-8 reagent (7Sea Biotech, Shanghai, China) was added to each well $\left(2 \times 10^{4} /\right.$ well $)$ in a 96 -well plate for $4 \mathrm{~h}$ at $37^{\circ} \mathrm{C}$, as described previously (17). Fluorescence intensity was determined using a Bio-Rad microplate reader (450 nm; Bio-Rad Laboratories, Inc., Hercules, CA, USA).

Osteogenic differentiation in vitro. For osteogenic differentiation, 3T3L1 cells were plated at a density of $5 \times 10^{4}$ cells/well in 12 -well plates for $24 \mathrm{~h}$ prior to induction. Osteogenic differentiation was induced by culturing the $3 \mathrm{~T} 3 \mathrm{~L} 1$ cells in osteogenic differentiation medium (ODM, DMEM supplemented with $20 \mathrm{mM} \beta$-glycerol phosphate, $50 \mu \mathrm{g} / \mathrm{ml}$ ascorbic acid and $100 \mathrm{nM}$ dexamethasone) for 21 days; the medium was replaced every 3-4 days (18). In order to determine the effects of the exosomes on osteogenic differentiation, Alizarin red staining (ARS) and reverse transcription-quantitative polymerase chain reaction (RT-qPCR) were carried out. In addition, $2 \mu \mathrm{g} / \mathrm{ml}$ 3T3L1-exo was added to the 3T3L1 cells in ODM for 15, 30, 60 and $120 \mathrm{~min}$ to determine its effects on differentiation.

RT-qPCR analysis of osteogenic gene expression. RT-qPCR was used to determine the expression levels of osteogenic differentiation-associated genes in 3T3L1 cells stimulated by 3T3L1-exo or pretreated with $10 \mu \mathrm{M}$ transforming growth factor- $\beta$ (TGF- $\beta 1$ ) inhibitor (SB431542) at $37^{\circ} \mathrm{C}$ for $30 \mathrm{~min}$, and miRNA expression in 3T3L1-exo or 3T3L1 cells. Briefly, RNA was isolated using TRIzol ${ }^{\circledR}$ reagent (Invitrogen; Thermo Fisher Scientific, Inc.) and cDNA was synthesized using PrimeScript ${ }^{\mathrm{TM}}$ RT Reagent kits (Takara Biotechnology Co., Ltd., Dalian, China) prior to qPCR, according manufacturer's protocol.

The expression levels of six pro-osteogenic genes were analyzed. The genes and primers used in the present study are listed in Table I. qPCR analysis of mRNA expression was performed using SYBR Primer Ex Taq ${ }^{\mathrm{TM}}$ II kits (Takara Biotechnology Co., Ltd.) under the following conditions: One 
Table I. Primers used for reverse transcription-quantitative polymerase chain reaction.

\begin{tabular}{lll}
\hline Gene & \multicolumn{1}{c}{ Forward $\left(5^{\prime}-3^{\prime}\right)$} & \multicolumn{1}{c}{ Reverse $\left(5^{\prime}-3^{\prime}\right)$} \\
\hline ALP & GAGCGTCATCCCAGTGGAG & TAGCGGTTACTGTAGACACCC \\
OCN & GAGGGCAATAAGGTAGTGAA & CATAGATGCGTTTGTAGGC \\
BSP & CAGGGAGGCAGTGACTCTTC & AGTGTGGAAAGTGTGGCGTT \\
RUNX2 & ATGCTTCATTCGCCTCACAAA & GCACTCACTGACTCGGTTGG \\
Osterix & GGAAAGGAGGCACAAAGAAGC & CCCCTTAGGCACTAGGAGC \\
Col I & CCCTGCCTGCTTCGTGTA & TTGAGTTTGGGTTGTTCGTC \\
-actin & CGTTGACATCCGTAAAGACC & AACAGTCCGCCTAGAAGCAC
\end{tabular}

ALP, alkaline phosphatase; BSP, bone sialoprotein; Col I, collagen-type I; OCN, osteocalcin; RNX2, runt-related transcription factor 2.

cycle at $95^{\circ} \mathrm{C}$ for $30 \mathrm{sec}$, followed by 40 cycles at $95^{\circ} \mathrm{C}$ for $5 \mathrm{sec}$ and $60^{\circ} \mathrm{C}$ for $34 \mathrm{sec}$. Primers for miRNA were purchased from iGeneBio (Guangzhou, China). qPCR analysis of miRNA expression was performed using All-in-One ${ }^{\mathrm{TM}}$ miRNA qRT-PCR Detection kit (GeneCopoeia, Inc., Rockville, MD, USA) under the following conditions: One cycle at $95^{\circ} \mathrm{C}$ for $10 \mathrm{~min}$, followed by 40 cycles at $95^{\circ} \mathrm{C}$ for $10 \mathrm{sec}, 60^{\circ} \mathrm{C}$ for $20 \mathrm{sec}$ and $72^{\circ} \mathrm{C}$ for $10 \mathrm{sec}$. Expression levels were quantified using the $2^{-\Delta \Delta \mathrm{Cq}}$ method (19). Data are presented as mean fold changes with respect to controls. Statistical significance was determined using Student's t-test or one-way analysis of variance (ANOVA) followed by Tukey's multiple comparisons test (11).

Western blot analysis. In accordance with a previously reported method (17), $10 \mu \mathrm{g}$ exosomes or crude proteins extracted from cell lysates were separated by $12 \%$ SDS-PAGE and transferred onto polyvinylidene fluoride membranes (EMD Millipore, Billerica, MA, USA). Membranes were blocked with 5\% milk in PBS-Tween and were then incubated with the primary antibodies $(1: 1,000)$ at $4^{\circ} \mathrm{C}$ overnight, followed by horseradish peroxidase-conjugated secondary antibodies (cat. nos. 7074 and 7076; 1:5,000; Cell Signaling Technology, Inc., Danvers, MA, USA) at room temperature for $1 \mathrm{~h}$. The membranes were scanned using a Tanon 4500 (Tanon Science and Technology Co., Ltd., Shanghai, China), according to the manufacturer's protocol. The following primary antibodies were used: Tumor susceptibility gene 101 (TSG101) mouse monoclonal antibody (mAb) (C-2; cat. no. sc-7964), heat shock protein $90 \beta$ family member 1 (GRP94) rabbit polyclonal antibody (H-212; cat. no. sc-11402), heat shock protein 70 (HSP70) mouse mAb (3A3; cat. no. sc-32239), $\beta$-actin (4E8H3; cat. no. sc-130065) mouse mAb (Santa Cruz Biotechnology, Inc., Dallas, TX, USA), SMAD family member 3 (smad3) rabbit mAb (C67H9), phosphorylated (p)-smad3 rabbit mAb (Ser423/425) (C25A9), and peroxisome proliferator-activated receptor- $\gamma$ (PPAR- $\gamma$ ) rabbit mAb (C26H12) (Cell Signaling Technology, Inc.).

Histological analysis. Osteogenic differentiation was detected using ARS on day 21, in order to quantify mineralization. For mineralization quantification, $40 \mathrm{mM}$ ARS (Sigma-Aldrich; Merck KGaA) was prepared in $\mathrm{dH}_{2} \mathrm{O}$ ( $\mathrm{pH} 4$.1). Cells (3T3L1; $1 \times 10^{5} /$ well) were cultured in ODM and $2 \mu \mathrm{g} / \mathrm{ml} 3 \mathrm{~T} 3 \mathrm{~L}$-exo for 21 days, rinsed three times with PBS, and fixed in $10 \%(\mathrm{v} / \mathrm{v})$ buffered neutral formalin (Sigma-Aldrich; Merck KGaA) for
$15 \mathrm{~min}$ at room temperature. The cells were then rinsed three times with $\mathrm{dH}_{2} \mathrm{O}$ and incubated at room temperature in ARS for $20 \mathrm{~min}$ with gentle agitation. Following the aspiration of unincorporated ARS, cells were rinsed four times with $\mathrm{dH}_{2} \mathrm{O}$. Images of stained cells were subsequently captured.

RNA sequencing of $3 T 3 L 1$ exosomes and $3 T 3 L 1$ cells. Total RNA was prepared from 3T3L1 cells and 3T3L1-exo using TRIzol (Invitrogen; Thermo Fisher Scientific, Inc.). RNA quantity was determined using an Agilent 2100 system (Agilent Technologies, Inc., Santa Clara, CA, USA). cDNA sequence libraries were established, sequenced and analyzed by Beijing Genomics Institute (Beijing, China) using the BGISEQ-500 sequencing technique (MGI Tech Co., Ltd., Shenzhen, China). Cluster analysis was performed using pheatmap in R software version 3.1.1 (www.r-project.org).

Electron microscopy. In accordance with a previously described method (17), exosome pellets were fixed in $4 \%$ paraformaldehyde at $4^{\circ} \mathrm{C}$ for $1 \mathrm{~h}$. The pellets were then loaded onto electron microscopy grids coated with Formvar carbon, contrasted, and embedded in a mixture of $2 \%$ uranyl acetate with methylcellulose. Sections were observed using a Philips Tecnai-10 transmission electron microscope operating at 80 kV (Phillips Electronic Instruments, Mahwah, NJ, USA).

Transient transfection of miR-223 mimics. To induce miR-223 overexpression, synthetic $100 \mathrm{nM}$ miRNA mimics were transfected into 3T3L1 cells (2x10 $/$ well) using $3 \mu$ INTERFERin ${ }^{\circledR}$ small interfering RNA transfection reagent (Polyplus-transfection SA, Illkirch, France) on a 24 -well plate at $37^{\circ} \mathrm{C}$ for $24 \mathrm{~h}$. The miR-223 mimic and NC mimic (Shanghai GenePharma Co., Ltd., Shanghai, China) sequences were as follows: miR-223 mimic forward, 5'-UGUCAGUUUGUCAAAUACCCA-3' and reverse, 5'-GGGUAUUUGACAAACUGACAUU-3'; NC mimics forward, 5'-UUCUCCGAACGUGUCACGUTT-3' and reverse, 5'-ACGUGACACGUUCGGAGAATT-3'.

Statistical analysis. Data are presented as the means \pm standard error of the mean. Data were analyzed by unpaired t-test or one-way analysis of variance followed by Tukey's post-hoc test using GraphPad Prism 7 software (GraphPad Software, Inc., La Jolla, CA, USA). P<0.05 was considered to indicate a statistically significant difference. 


\section{Results}

Isolation and identification of 3T3L1-exo. Exosomes were isolated from 3T3L1 cells cultured under normal growth conditions (3T3L1-exo). Exosome size and morphology were determined using electron microscopy. Exosomes were revealed to range between 50 and $100 \mathrm{~nm}$ in size, and exhibited a typical rounded shape (Fig. 1A). Like their parent 3T3L1 cells, 3T3L1-exo was revealed to express specific markers, including TSG101 and HSP70, whereas GRP94, endoplasmic reticulum-residing protein, was absent in 3T3L1-exo (Fig. 1B). Similar to their cell counterparts, 3T3L1-exo did not express the adipogenic transcription factor PPAR- $\gamma$; however, differentiated 3T3L1 cells did express PPAR- $\gamma$ (Fig. 1C). These results confirmed the integrity of 3T3L1-exo.

3T3L1-exo has no effect on 3T3L1 preadipocyte proliferation and apoptosis. To determine the effects of 3T3L1-exo on 3T3L1 cell survival, 3T3L1 preadipocyte proliferation and apoptosis were detected following stimulation by 3T3L1-exo. Exosomes did not affect 3T3L1 cell proliferation, even with an increased concentration (Fig. 2A). Comparably, 3T3L1-exo had no effect on the apoptosis of 3T3L1 cells (Fig. 2B).

3T3L1-exo mediates $3 T 3 L 1$ preadipocyte osteogenic differentiation. Since 3T3L1-exo had no effect on 3T3L1 cell survival, and because MSC-exo has been reported to mediate osteogenic differentiation of MSCs, preadipocytes and mature osteoblasts (11), the present study determined whether 3T3L1-exo promoted the osteogenic differentiation of 3T3L1 cells (1). 3T3L1 cells were stimulated by 3T3L1-exo in ODM; subsequently, the cells were stained with ARS and the expression levels of pro-osteogenic genes were analyzed by RT-qPCR. As shown in Fig. 3A and B, osteogenic differentiation was enhanced in 3T3L1 cells stimulated by 3T3L1-exo in ODM. Furthermore, the expression levels of pro-osteogenic genes, including alkaline phosphatase, osteocalcin, bone sialoprotein, osterix, collagen-type I and runt-related transcription factor 2 (RUNX2), were all increased in 3T3L1 cells exposed to ODM and 3T3L1-exo.

3T3L1-exo activates $3 T 3 L 1$ preadipocytes to undergo osteogenic differentiation via TGF- $\beta$ signaling. The present findings suggested that 3T3L1-exo promoted the osteogenic differentiation of 3T3L1 cells. RUNX2 activates and regulates osteogenesis and acts as a target gene for numerous signaling pathways, including TGF- $\beta 1$, bone morphogenetic protein, Wnt, Hedgehog and Nel-like protein type-1 (5). The present study demonstrated that exposure of cells to 3T3L1-exo induced Smad3 phosphorylation (Fig. 4A). Subsequently, the mRNA expression levels of RUNX2 were detected, and it was revealed that there was no significant difference between the ODM + exo and ODM groups when TGF- $\beta$ was inhibited through a Smad3 inhibitor (SB431542; Beyotime Institute of Biotechnology, Shanghai, China) (Fig. 4B). These results indicated that 3T3L1-exo may promote 3T3L1 cell osteogenic differentiation via the TGF- $\beta 1$ pathway.

miR-223 in 3T3L1-exo may be involved in enhanced osteogenic differentiation of $3 T 3 L 1$ preadipocytes. To
A

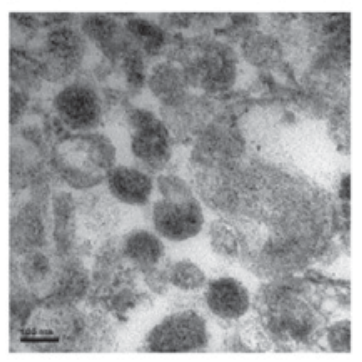

B
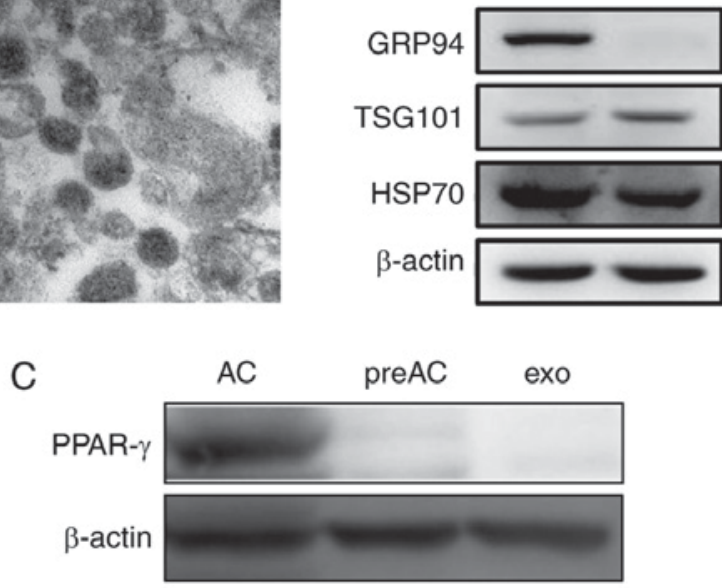

Figure 1. Isolation and identification of 3T3L1-exo. (A) Electron micrograph of 3T3L1-exo. Scale bar, $100 \mathrm{nM}$. (B) Western blot analysis of exosome markers. (C) Western blot analysis of the adipogenic marker PPAR- $\gamma$. AC, differentiated 3T3L1 cells; exo/3T3L1-exo, 3T3L1 cell derived-exosomes; GRP94, heat shock protein $90 \beta$ family member 1 ; HSP70, heat shock protein 70; PPAR- $\gamma$, peroxisome proliferator-activated receptor- $\gamma$; preAC, untreated 3T3L1 cells; TSG101, tumor susceptibility gene 101

explore the mechanism by which 3T3L1-exo promoted 3T3L1 osteogenic differentiation, 3T3L1 cells and 3T3L1-exo were sequenced to determine miRNA profiles, and a similarly wide distribution profile of read lengths was confirmed (Fig. 5A), with predominant peaks at 20-24 nucleotides and 17-20 nucleotides, respectively. Since miRNAs are critical regulators of signaling pathways, miRNA expression patterns were compared between exosomes and cells, and it was revealed that 427 miRNAs were upregulated and 573 were downregulated in the exosomes compared with in the cells (Fig. 5B). To confirm the differences and relationships of miRNAs derived from cells and exosomes, cluster analysis using pheatmap in R software was performed (Fig. 5C).

In the present study, miR-223 was highlighted as a potential candidate target; this miRNA is a key regulatory factor in osteoclast and osteoblast differentiation (20). Therefore, RT-qPCR was performed to confirm the expression of miR-223 in the 3T3L1-exo and 3T3L1 cells; miR-223 and miR-451a was revealed to be upregulated in 3T3L1-exo compared with in 3T3L1 cells (Fig. 5D). Furthermore, miR-223 expression was detected in 3T3L1 cells stimulated with ODM and/or 3T3L1-exo for 48 and $72 \mathrm{~h}$. Notably, miR-223 was downregulated in ODM + 3T3L1-exo stimulated cells compared with in cells stimulated with ODM alone (Fig. 5E). It was therefore hypothesized that 3T3L1-exo may suppress miR-223 expression in 3T3L1 preadipocytes through a competitive mechanism or via another miRNA, and that other factors may regulate the decreased levels of miR-223. The results of a miR-223 RNA interference experiment (Fig. 5F) demonstrated that miR-223 mimics weakened the increased expression of RUNX2 in 3T3L1 cells exposed to ODM and 3T3L1-exo (Fig. 5G). These results suggested that miR-223 in 3T3L1-exo may be involved in enhanced osteogenic differentiation of 3T3L1 preadipocytes. 

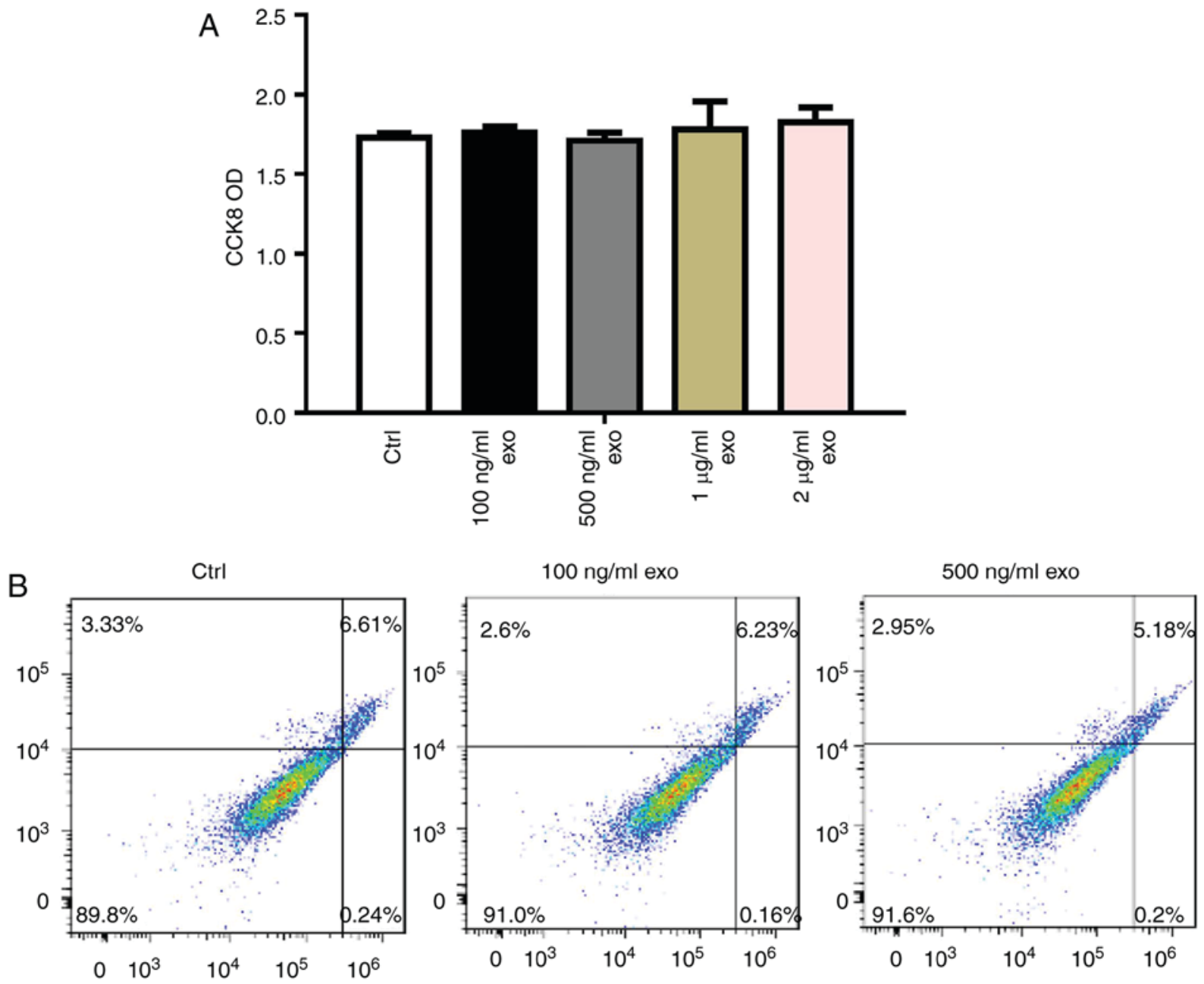

$1 \mu \mathrm{g} / \mathrm{ml}$ exo
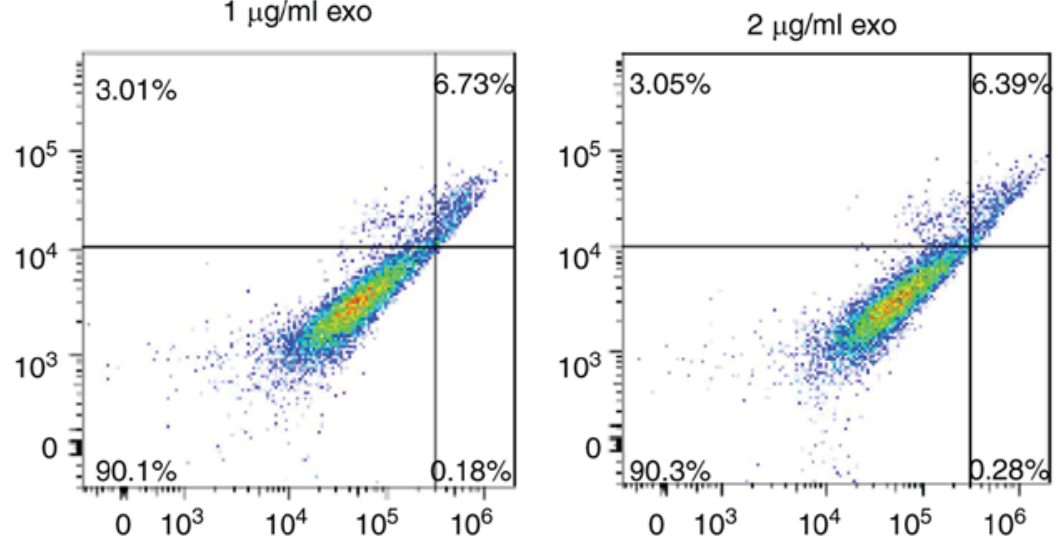

Figure 2. 3T3L1-exo has no effect on 3T3L1 preadipocyte proliferation and apoptosis. (A) CCK8 was used to determine the effects of 3T3L1-exo on 3T3L1 cell proliferation (all groups, $\mathrm{P}=0.9132$ ). (B) Fluorescence-activated cell sorting analysis was used to detect the effects of 3T3L1-exo on 3T3L1 cell apoptosis The percentages represent the percentage of cells in each quadrant. CCK8, Cell Counting kit-8; Ctrl, control; exo/3T3L1-exo, 3T3L1 cell derived-exosomes; OD, optical density.

\section{Discussion}

Clinical bone implants often require bone fillers or enhanced regeneration due to a shortage of bone. In dentistry, a significant proportion of patients that need implants require increased bone volume prior to implant placement. The clinical gold standard for bone grafting is an autograft; however, this method has limitations, including donor-site morbidity, limited availability of grafting material and compromised bone quality in patients with osteoporosis (4). Aside from autografts, guided bone regeneration using a specially selected bovine source is the most straightforward procedure for bone transplantation; however, this method can result in rejection and insufficient osteogenesis (3).

Bone regeneration requires the migration of specific cells to the healing site to proliferate there and to provide a biological substrate for new tissue growth. BMSCs have the ability to form bone; therefore, bone marrow transplantation is used 


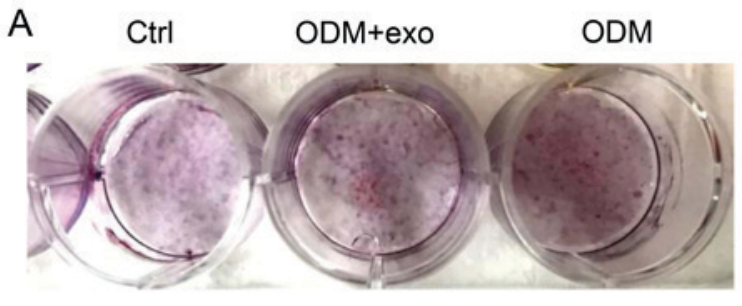

B

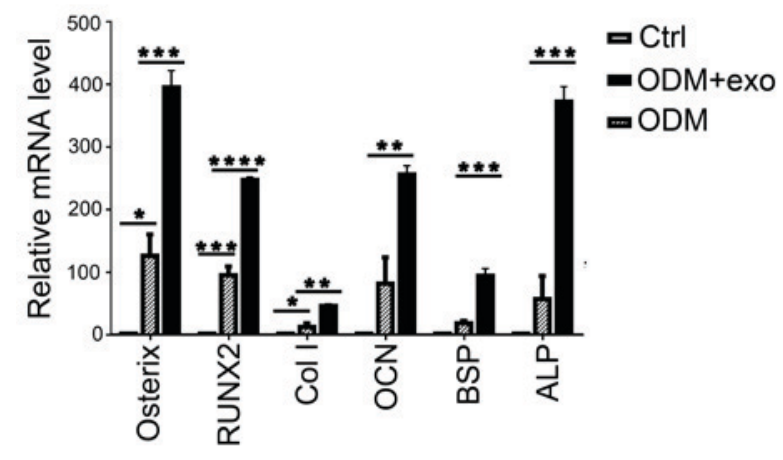

Figure 3. 3T3L1-exo mediates 3T3L1 preadipocyte osteogenic differentiation. (A) ARS staining was conducted to determine the effects of 3T3L1-exo on 3T3L1 preadipocyte osteogenic differentiation. (B) Reverse transcription-quantitative polymerase chain reaction was performed to determine the effects of 3T3L1-exo on the expression of osteogenic differentiation-associated genes. ${ }^{*} \mathrm{P}<0.05,{ }^{* *} \mathrm{P}<0.01,{ }^{* * *} \mathrm{P}<0.001,{ }^{* * * *} \mathrm{P}<0.0001$ (ODM + exo vs. ODM: osterix, $\mathrm{P}=0.0007$; RUNX2, $\mathrm{P}<0.0001$; Col I, $\mathrm{P}=0.0022$; OCN, $\mathrm{P}=0.007$; $\mathrm{BSP}, \mathrm{P}=0.0008$; ALP, $\mathrm{P}=0.0003$, ctrl vs. ODM: osterix, $\mathrm{P}=0.0298$; RUNX2, $\mathrm{P}=0.0006$; $\mathrm{Col} \mathrm{I}, \mathrm{P}=0.024$; $\mathrm{OCN}, \mathrm{P}=0.05$; BSP, $\mathrm{P}=0.2927$; ALP, $\mathrm{P}=0.3391$ ). ALP, alkaline phosphatase; $\mathrm{BSP}$, bone sialoprotein; Col I, collagen-type I; Ctrl, control; exo/3T3L1-exo, 3T3L1 cell derived-exosomes; OCN, osteocalcin; ODM, osteogenic differentiation medium; RNX2, runt-related transcription factor 2.

clinically in combination with osteoconductive materials to augment bone healing (21). However, BMSCs are limited with regards to insufficiency in numbers. Research has indicated that ADSCs are a more suitable tissue source (1), since they are also capable of undergoing osteogenic differentiation but are more readily obtained.

Because the numbers of stem cells are limited, agents that promote their differentiation are required. To promote bone formation, specific growth factors are often applied. However, research has suggested that the secreted trophic factors are more important than the process of stem cell differentiation in mediating therapeutic efficacy. The exosome, a secreted membrane vesicle, is therefore an active therapeutic factor in the process of MSC secretion (22).

In the present study, 3T3L1-exo was revealed to promote 3T3L1 preadipocyte osteogenic differentiation via the TGF- $\beta$ pathway. Notably, the TGF- $\beta$ pathway can activate RUNX2 and further induce the osteogenic differentiation of cells (5). Furthermore, miRNA sequences were detected in 3T3L1 cells and 3T3L1-exo, and it was revealed that, in some cases, miRNAs were comparable between cells and exosomes; however, in other cases, miRNAs were expressed at a higher level in exosomes.

MSCs can promote TGF- $\beta$ expression in murine renal tubular epithelial cells via miR-223 (23). Furthermore, bone marrow-derived miR-223 has an effect on vascular endothelial
A

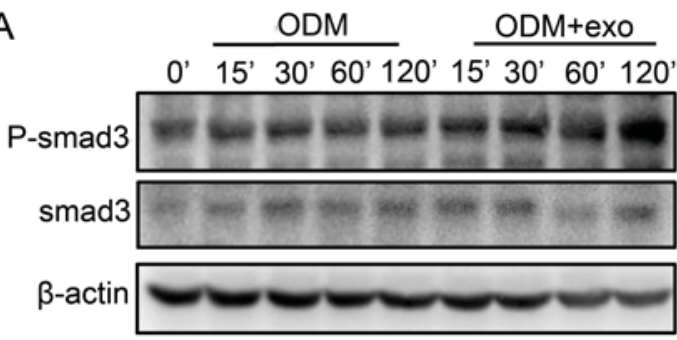

B

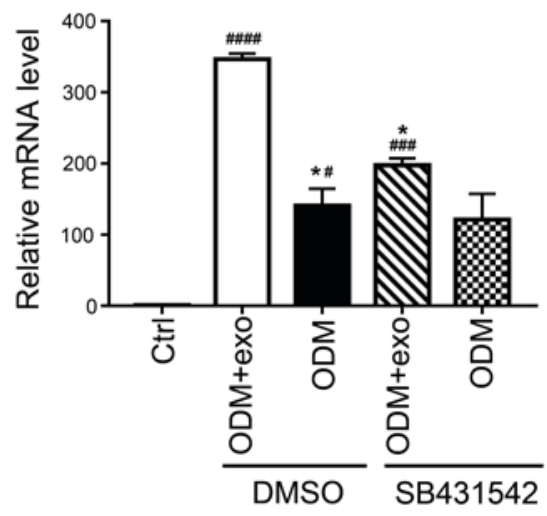

Figure 4. 3T3L1-exo activates 3T3L1 preadipocytes to undergo osteogenic differentiation via TGF- $\beta$ signaling. (A) Western blot analysis detected p-smad3 and smad3 expression in 3T3L1 cells following stimulation with 3T3L1-exo for the indicated durations. (B) Reverse transcription-quantitative polymerase chain reaction was conducted to determine the mRNA expression levels of runt-related transcription factor 2 in 3T3L1 cells stimulated with ODM and/or 3T3L1 exo following treatment with the transforming growth factor- $\beta$ inhibitor (SB431542). ${ }^{*} \mathrm{P}<0.05$ vs. DMSO+ODM+exo; ${ }^{\#} \mathrm{P}<0.05,{ }^{\# \# \#} \mathrm{P}<0.001,{ }^{\# \# \# \#} \mathrm{P}<0.0001$ vs. control. $(\mathrm{ODM}+$ exo vs. ODM: DMSO, $\mathrm{P}=0.0204 ; \mathrm{SB} 431542, \mathrm{P}=0.2204$; Ctrl vs. DMSO+ODM+exo, $\mathrm{P}<0.0001$; Ctrl vs. DMSO+ODM, $\mathrm{P}=0.0285$; Ctrl vs. SB431542+ODM+exo, P=0.0009; Ctrl vs. SB431542+ODM, $\mathrm{P}=0.0883$; DMSO+ODM+exo vs. SB431542+ODM+exo, $\mathrm{P}=0.0133$; DMSO+ODM vs. SB431542+ODM, P=0.9662). Ctrl, control; DMSO, dimethyl sulfoxide; exo/3T3L1-exo, 3T3L1 cell derived-exosomes; ODM, osteogenic differentiation medium; P, phosphorylated; Smad3, SMAD family member 3 .

cells as an endocrine genetic signal, and is involved in vascular injury by targeting insulin-like growth factor 1 receptor (24). In addition, Notch/miR-223 has been reported to modify the osteogenic potential of bone marrow stromal cells (25). Consequently, miR-223 may serve a regulatory role in 3T3L1-exo by enhancing the osteogenic differentiation of 3T3L1 cells.

The present study confirmed that the expression of miR-223 was increased in 3T3L1-exo compared with in 3T3L1 cells. Notably, the expression levels of miR-223 were decreased in 3T3L1 preadipocytes cultured in ODM and stimulated by 3T3L1-exo compared with in cells without exosome stimulation. It may be hypothesized that 3T3L1-exo suppresses the expression of miR-223 in 3T3L1 preadipocytes through a competitive mechanism, or by another miRNA, or a factor regulated by decreased miR-223. However, these competitive mechanisms or other regulated mechanisms require further investigation In conclusion, the application of 3T3L1-exo may be useful for investigating preadipocyte-induced bone regeneration. 
A
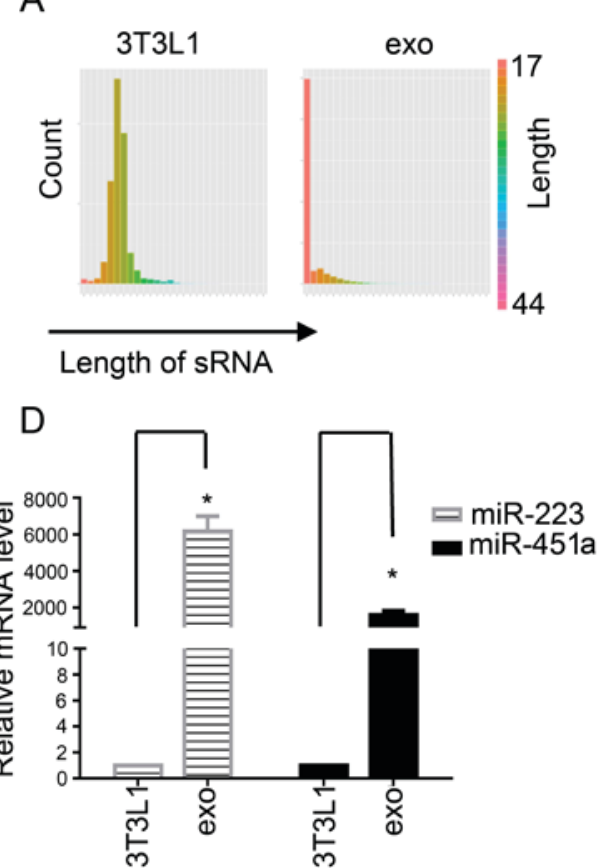

miR-223

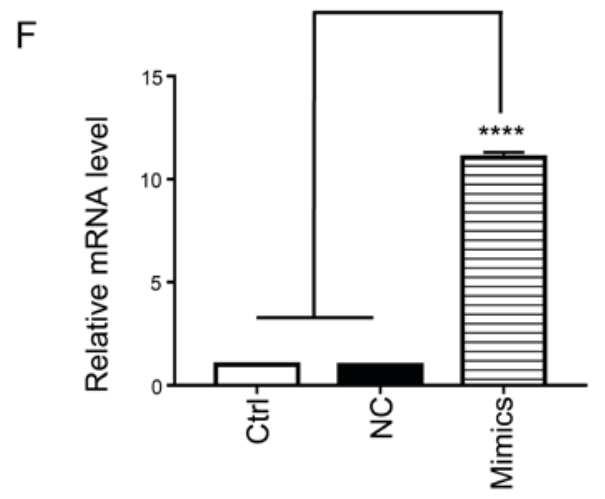

B

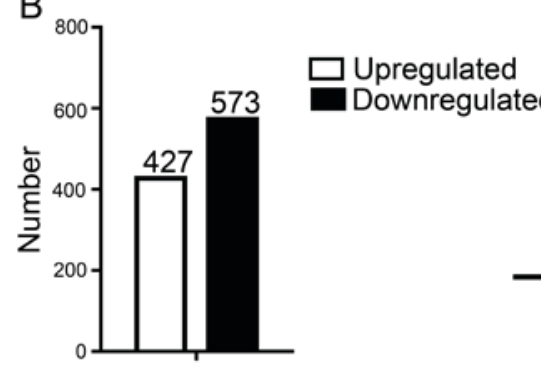

E

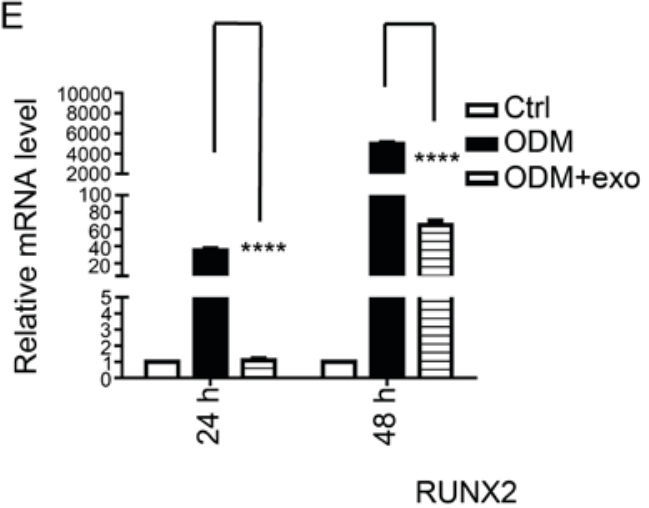

G
C

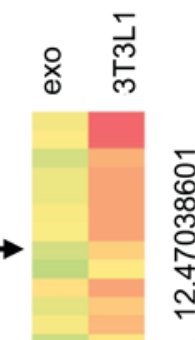

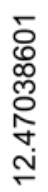

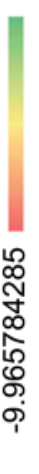

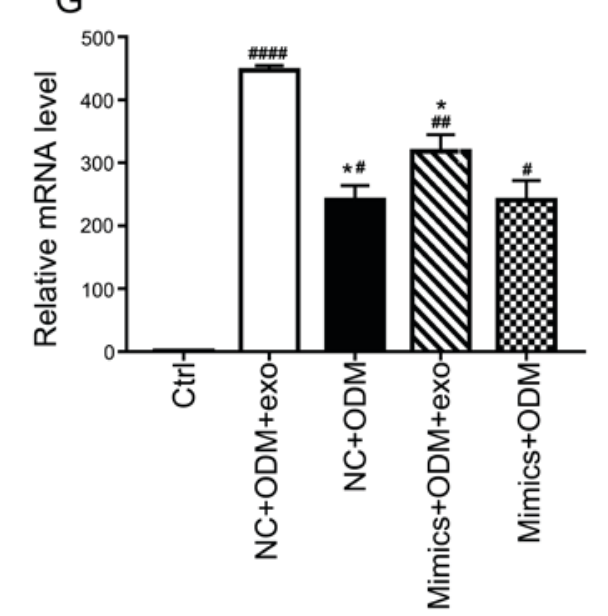

Figure 5. 3T3L1 cells and their exosomes differ in miRNA composition. (A) Distribution of the length of miRNA reads in 3T3L1 cells and 3T3L1-exo. (B) Differentially expressed miRNAs ( $\log 2$ ratio: 3T3L1-exo/3T3L1 cells). (C) Heat maps indicating the expression of the top 20 upregulated (left) and downregulated (right) miRNAs in 3T3L1-exo compared with in 3T3L1 cells. (D) RT-qPCR analysis revealed differences in miR-223 and miR-451a expression levels in 3T3L1 cells and 3T3L1-exo ${ }^{*} \mathrm{P}<0.05$ vs. 3T3L1 cells. (3T3L1 cells vs. 3T3L1 exo: miR-223, P=0.0185; miR-451a, P=0.0202). (E) RT-qPCR analysis was used to detect the expression levels of miR-223 in 3T3L1 preadipocytes stimulated with ODM and/or 3T3L1-exo for 48 and $72 \mathrm{~h}$. **** $\mathrm{P}<0.0001 \mathrm{vs.}$ ODM. (ODM + exo vs. ODM: 48 h, P<0.0001; 72 h, P<0.0001). (F) RT-qPCR was used to test the efficacy of miR-223 mimic transfection (mimics vs. ctrl: P<0.0001; mimics vs. NC: P<0.0001). (G) RT-qPCR was used to detect the mRNA expression levels of runt-related transcription factor 2 in 3 T3L1 cells stimulated with ODM and/or 3T3L1 exo post-transfection with miR-223 mimics. ${ }^{*} \mathrm{P}<0.05$ vs. NC+ODM+exo; ${ }^{\#} \mathrm{P}<0.05,{ }^{\# \#<0.01, ~}{ }^{\# \# \# "} \mathrm{P}<0.0001$ vs. control. (ODM+exo vs. ODM: $\mathrm{NC}, \mathrm{P}=0.0200 ;$ mimics, $\mathrm{P}=0.1578 ; \mathrm{NC}+\mathrm{ODM}+$ exo vs. mimics+ODM+exo, $\mathrm{P}=0.0322 ; \mathrm{NC}+\mathrm{ODM}$ vs. mimics+ODM, $\mathrm{P}>0.9999 ; \mathrm{Ctrl}$ vs. $\mathrm{NC}+\mathrm{ODM}+\mathrm{exo}$, $\mathrm{P}<0.0001$; Ctrl vs. NC+ODM, $\mathrm{P}=0.01$; Ctrl vs. mimics+ODM+exo, $\mathrm{P}=0.0079$; ctrl vs mimics+ODM, P=0.0173). Ctrl, control; exo/3T3L1-exo, 3T3L1 cell derived-exosomes; miR/miRNA, microRNA; NC, negative control; ODM, osteogenic differentiation medium; sRNA, small RNA.

\section{Acknowledgments}

The authors would like to thank Dr Elizabeth Finnie for editing the English text of a draft of this manuscript.

\section{Funding}

The present study was supported by grants from the National Natural Science Foundation of China (grant no. 81700972).

\section{Availability of data and materials}

The datasets used and/or analyzed during the current study are available from the corresponding author on reasonable request.

\section{Authors' contributions}

HW conceived and designed the study; WD, LS and NZ performed the experiments; and WD and LS completed the draft. All authors read and approved the manuscript. 


\section{Ethics approval and consent to participate}

Not applicable.

\section{Patient consent for publication}

Not applicable.

\section{Competing interests}

The authors declare that they have no competing interests.

\section{References}

1. Bressan E, Botticelli D, Sivolella S, Bengazi F, Guazzo R, Sbricoli L, Ricci S, Ferroni L, Gardin C, Velez JU and Zavan B: Adipose-derived stem cells as a tool for dental implant osseointegration: An experimental study in the dog. Int J Mol Cell Med 4 197-208, 2015

2. Padial-Molina M, O'Valle F, Lanis A, Mesa F, Dohan Ehrenfest DM, Wang HL and Galindo-Moreno P: Clinical application of mesenchymal stem cells and novel supportive therapies for oral bone regeneration. Biomed Res Int 2015 341327, 2015.

3. Théry C, Zitvogel L and Amigorena S: Exosomes: Composition, biogenesis and function. Nat Rev Immunol 2: 569-579, 2002.

4. Grayson WL, Bunnell BA, Martin E, Frazier T, Hung BP and Gimble JM: Stromal cells and stem cells in clinical bone regeneration. Nat Rev Endocrinol 11: 140-150, 2015.

5. James AW: Review of signaling pathways governing MSC osteogenic and adipogenic differentiation. Scientifica (Cairo) 2013 684736, 2013.

6. Qin Y, Sun R, Wu C, Wang L and Zhang C: Exosome: A novel approach to stimulate bone regeneration through regulation of osteogenesis and angiogenesis. Int J Mol Sci 17: E712, 2016.

7. Baglio SR, Rooijers K, Koppers-Lalic D, Verweij FJ, Pérez Lanzón M, Zini N, Naaijkens B, Perut F, Niessen HW, Baldini $\mathrm{N}$ and Pegtel DM: Human bone marrow- and adipose-mesenchymal stem cells secrete exosomes enriched in distinctive miRNA and tRNA species. Stem Cell Res Ther 6 : $127,2015$.

8. Gernapudi R, Yao Y, Zhang Y, Wolfson B, Roy S, Duru N, Eades G, Yang P and Zhou Q: Targeting exosomes from preadipocytes inhibits preadipocyte to cancer stem cell signaling in early-stage breast cancer. Breast Cancer Res Treat 150: 685-695, 2015.

9. Fleury A, Martinez MC and Le Lay S: Extracellular vesicles as therapeutic tools in cardiovascular diseases. Front Immunol 5: 370, 2014.

10. Yu B, Zhang X and Li X: Exosomes derived from mesenchymal stem cells. Int J Mol Sci 15: 4142-4157, 2014.

11. Narayanan R, Huang CC and Ravindran S: Hijacking the cellular mail: Exosome mediated differentiation of mesenchymal stem cells. Stem Cells Int 2016: 3808674, 2016.
12. Cui Y, Luan J, Li H, Zhou X and Han J: Exosomes derived from mineralizing osteoblasts promote ST2 cell osteogenic differentiation by alteration of microRNA expression. FEBS Lett 590: 185-192, 2016.

13. Wang Z, Ding L, Zheng XL, Wang HX and Yan HM: DC-derived exosomes induce osteogenic differentiation of mesenchymal stem cells. Zhongguo Shi Yan Xue Ye Xue Za Zhi 22: 600-604, 2014 (In Chinese).

14. Ekström K, Omar O, Granéli C, Wang X, Vazirisani F and Thomsen P: Monocyte exosomes stimulate the osteogenic gene expression of mesenchymal stem cells. PLoS One 8: e75227, 2013.

15. Yeo RW, Lai RC, Zhang B, Tan SS, Yin Y, Teh BJ and Lim SK: Mesenchymal stem cell: An efficient mass producer of exosomes for drug delivery. Adv Drug Deliv Rev 65: 336-341, 2013.

16. Lopatina T, Bruno S, Tetta C, Kalinina N, Porta M and Camussi G: Platelet-derived growth factor regulates the secretion of extracellular vesicles by adipose mesenchymal stem cells and enhances their angiogenic potential. Cell Commun Signal 12: 26 , 2014.

17. Cai Z, Yang F, Yu L, Yu Z, Jiang L, Wang Q, Yang Y, Wang L, Cao $\mathrm{X}$ and Wang J: Activated T cell exosomes promote tumor invasion via Fas signaling pathway. J Immunol 188: 5954-5961, 2012.

18. Wan DC, Shi YY, Nacamuli RP, Quarto N, Lyons KM and Longaker MT: Osteogenic differentiation of mouse adipose-derived adult stromal cells requires retinoic acid and bone morphogenetic protein receptor type IB signaling. Proc Natl Acad Sci USA 103: 12335-12340, 2006.

19. Livak KJ and Schmittgen TD: Analysis of relative gene expression data using real-time quantitative PCR and the 2(-Delta Delta C(T)) method. Methods 25: 402-408, 2001

20. Guan X, Gao Y, Zhou J, Wang J, Zheng F, Guo F, Chang A, Li $X$ and Wang B: miR-223 regulates adipogenic and osteogenic differentiation of mesenchymal stem cells through a C/EBPs/miR-223/FGFR2 regulatory feedback loop. Stem Cells 33: 1589-1600, 2015.

21. Marolt D, Knezevic M and Novakovic GV: Bone tissue engineering with human stem cells. Stem Cell Res Ther 1: 10, 2010.

22. Lai RC, Yeo RW, Tan SS, Zhang B, Yin Y, Kwan Sze NS, Choo A and Lim SK: Mesenchymal stem cell exosomes: The future MSC-based therapy? Mesench Stem Cell Ther: 39-61, 2012.

23. Yuan X, Wang X, Chen C, Zhou J and Han M: Bone mesenchymal stem cells ameliorate ischemia/reperfusion-induced damage in renal epithelial cells via microRNA-223. Stem Cell Res Ther 8: 146, 2017.

24. Chu M, Wu R, Qin S, Hua W, Shan Z, Rong X, Zeng J, Hong L, Sun Y, Liu Y, et al: Bone marrow-derived MicroRNA-223 works as an endocrine genetic signal in vascular endothelial cells and participates in vascular injury from kawasaki disease. J Am Heart Assoc 6: e004878, 2017.

25. Berenstein R, Nogai A, Waechter M, Blau O, Kuehnel A, Schmidt-Hieber M, Kunitz A, Pezzutto A, Dörken B and Blau IW: Multiple myeloma cells modify VEGF/IL-6 levels and osteogenic potential of bone marrow stromal cells via Notch/miR-223. Mol Carcinog 55: 1927-1939, 2016.

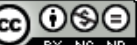

This work is licensed under a Creative Commons Attribution-NonCommercial-NoDerivatives 4.0 International (CC BY-NC-ND 4.0) License. 